\title{
Less invasive surgery for treating adult spinal deformities: ceiling effects for deformity correction with 3 different techniques
}

\author{
Michael Y. Wang, M.D., ${ }^{1}$ Praveen V. Mummaneni, M.D., ${ }^{2}$ Kai-Ming G. Fu, M.D., Ph.D., ${ }^{3}$ \\ Neel Anand, M.D.,${ }^{4}$ David O. OKonkwo, M.D., Ph.D.,${ }^{5}$ Adam S. Kanter, M.D., 5 \\ Frank La Marca, M.D., ${ }^{6}$ Richard Fessler, M.D., Ph.D., ${ }^{7}$ Juan Uribe, M.D., ${ }^{8}$ \\ Christopher I. Shaffrey, M.D., ${ }^{9}$ Virginie Lafage, Ph.D., ${ }^{10}$ RaQeeb M. HaQue, M.D., ${ }^{11}$ \\ Vedat Deviren, M.D., ${ }^{2}$ and Gregory M. Mundis JR., M.D., ${ }^{12}$ ON Behalf of The Minimally \\ Invasive Surgery Section of the International Spine Study Group
}

\begin{abstract}
${ }^{1}$ University of Miami, Neurosurgery, Miami, Florida; ${ }^{2}$ University of California, Department of Neurosurgery, San Francisco, California; ${ }^{3}$ Weill Cornell Medical College, Department of Neurosurgery, New York, New York; ${ }^{4}$ Cedars-Sinai Hospital, Department of Neurosurgery, Los Angeles, California; ${ }^{5}$ University of Pittsburgh Medical Center, Department of Neurological Surgery, Pittsburgh, Pennsylvania; ${ }^{6}$ University of Michigan, Neurosurgery, Ann Arbor, Michigan; ${ }^{7}$ Rush University Medical Center, Department of Neurosurgery, Chicago, Illinois; ${ }^{8}$ University of South Florida, Neurosurgery, Tampa, Florida; ${ }^{9}$ University of Virginia Medical Center, Department of Neurological Surgery, Charlottesville, Virginia; ${ }^{10}$ NYU Langone Medical Center, Orthopaedic Surgery, New York, New York; ${ }^{11}$ Columbia University, Neurosurgery, New York, New York; and ${ }^{12}$ San Diego Center for Spinal Disorders, Orthopaedic Surgery, San Diego, California
\end{abstract}

Object. Minimally invasive surgery (MIS) options for the treatment of adult spinal deformity (ASD) have advanced significantly over the past decade. However, a wide array of options have been described as being MIS or less invasive. In this study the authors investigated a multiinstitutional cohort of patients with ASD who were treated with less invasive methods to determine the extent of deformity correction achieved.

Methods. This study was a retrospective review of multicenter prospectively collected data in 85 consecutive patients with ASD undergoing MIS surgery. Inclusion criteria were as follows: age older than 45 years; minimum $20^{\circ}$ coronal lumbar Cobb angle; and 1 year of follow-up. Procedures were classified as follows: 1$)$ stand-alone $(\mathrm{n}=7) ; 2)$ circumferential MIS $(\mathrm{n}=43)$; or 3 ) hybrid $(\mathrm{n}=35)$.

Results. An average of 4.2 discs (range 3-7) were fused, with a mean follow-up duration of 26.1 months in this study. For the stand-alone group the preoperative Cobb range was $22^{\circ}-51^{\circ}$, with $57 \%$ greater than $30^{\circ}$ and $28.6 \%$ greater than $50^{\circ}$. The mean Cobb angle improved from $35.7^{\circ}$ to $30^{\circ}$. A ceiling effect of $23^{\circ}$ for curve correction was observed, regardless of preoperative curve severity. For the circumferential MIS group the preoperative Cobb range was $19^{\circ}-62^{\circ}$, with $44 \%$ greater than $30^{\circ}$ and $5 \%$ greater than $50^{\circ}$. The mean Cobb angle improved from $32^{\circ}$ to $12^{\circ}$. A ceiling effect of $34^{\circ}$ for curve correction was observed. For the hybrid group the preoperative Cobb range was $23^{\circ}-82^{\circ}$, with $74 \%$ greater than $30^{\circ}$ and $23 \%$ greater than $50^{\circ}$. The mean Cobb angle improved from $43^{\circ}$ to $15^{\circ}$. A ceiling effect of $55^{\circ}$ for curve correction was observed.

Conclusions. Specific procedures for treating ASD have particular limitations for scoliotic curve correction. Less invasive techniques were associated with a reduced ability to straighten the spine, particularly with advanced curves. These data can guide preoperative technique selection when treating patients with ASD.

(http://thejns.org/doi/abs/10.3171/2014.3.FOCUS1423)

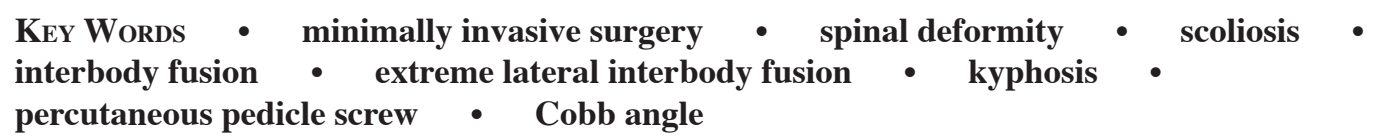

$\mathrm{S}$ URGERY for adult spinal deformity (ASD) remains a challenging proposition. Several factors contribute to create a high likelihood of intraoperative and

\footnotetext{
Abbreviations used in this paper: ASD = adult spinal deformity; ISSG = International Spine Study Group; MIS = minimally invasive surgery; NPS = Numeric Pain Score; ODI = Oswestry Disability Index; SVA = sagittal vertical axis; TLIF = transforaminal lumbar interbody fusion.
}

postoperative complication rates. Medical comorbidities, patient deconditioning due to pain and immobility, associated osteoporosis, a rigid skeletal deformity, and abnormal spinal anatomy all increase the likelihood of a complication from ASD surgery ${ }^{6,9}$ Furthermore, the surgical enterprise needed to destabilize, realign, and fuse the spine over multiple segments is painful and debilitating, requiring prolonged anesthesia and lengthy hospital stays. It is therefore not surprising that even case series 
from centers specializing in these operations report a high rate of serious complications. In a series of 361 patients reported by Pateder et al.,${ }^{10}$ the 30 -day mortality rate was $2.4 \%$. A more recent report by Smith et al. ${ }^{13}$ in which data from the Spinal Deformity Study Group were analyzed showed that $26.2 \%$ of 206 patients suffered a minor complication and $15.5 \%$ suffered a major complication.

Over the past decade spine surgeons have attempted to use the potential benefits of minimally invasive surgery (MIS) to address spinal deformities. ${ }^{1,11,15}$ For short-segment surgeries it appears that MIS techniques can result in less blood loss, lower infection rates, and quicker mobilization. If these principles could be applied to ASD surgery, then many of the problems associated with traditional open surgery could potentially be mitigated. ${ }^{12}$ Thus, a variety of techniques have evolved in an attempt to apply MIS methodologies to correct coronal spinal deformities.

To date, the plethora of techniques emerging to potentially reduce the morbidity of ASD surgery has only been described in reports focusing on single methods, without comparisons being made between the various techniques. In an effort to better understand the benefits and limitations of such approaches the International Spine Study Group (ISSG) ${ }^{3}$ formed a multiinstitutional effort to investigate MIS for ASD. The study group, composed of investigators from 9 academic medical centers, each with well-established expertise in MIS for ASD, assembled a retrospective cohort of patients to investigate the utility of MIS in the setting of ASD.

\section{Methods}

\section{The MIS-ISSG Patient Selection Criteria}

A total of 9 academic medical centers (Cedars Sinai, Northwestern University, University of California at San Diego, University of California at San Francisco [Neurosurgery], University of California at San Francisco [Orthopedics], University of Miami, University of Michigan, University of Pittsburgh, and University of South Florida) with well-established expertise in MIS for ASD were selected to participate in the MIS-ISSG. All centers obtained local institutional review board approval for participation in this study.

For this retrospective study each center collected data on a minimum of 10 and a maximum of 20 patients who had been consecutively treated with MIS techniques for ASD. The primary surgical procedure of interest had to have been performed after November 2009, and patients had to have a minimum of 12 months of clinical and radiographic follow-up. In addition, the following data were obtained in all patients at baseline (preoperative) and last follow-up: 36-in anteroposterior and lateral standing scoliosis radiographs, Oswestry Disability Index (ODI) score ${ }^{5}$ and separate Numeric Pain Scores (NPSs) for leg and back pain. The NPS was assessed on a 10-point scale. In addition, the database included patient demographic data (age, sex, body mass index, smoking status, previous spine surgeries, and American Society of Anesthesiologists grade); data for surgical parameters (total operating time, any staging of procedures, total blood loss, surgical methodology, number of levels treated, and routes of approach); and clinical outcomes (length of hospital stay, any blood transfusions, and major/minor complications allocated by subtype).

For inclusion in the database patients had to be more than 45 years old at the time of surgery. Their standing radiographs had to have either a coronal Cobb angle of greater than or equal to $20^{\circ}$ or a sagittal vertical axis (SVA) greater than $5 \mathrm{~cm}$. The coronal Cobb angle was determined by measuring the maximal coronal angulation between the 2 most angulated upper vertebral endplates on 36-in standing radiographs.

Lumbar lordosis was measured between the upper endplates of L-1 and S-1 in the standing lateral position with 36-in radiographs. The SVA was measured by dropping a plumb line from the anterior inferior aspect of the C-7 vertebra. The minimum distance from this line to the posterior superior endplate of S-1 was the SVA measured in centimeters. Data from all centers were combined in Excel, and data analysis was performed using SPSS software.

\section{Surgical Techniques: MIS for ASD}

Due to the diversity of specific techniques used to treat complex and varied pathological conditions in ASD, consensus was needed on how to classify the surgical procedures. Based on MIS-ISSG consensus meetings the procedures were categorized into 1 of 3 types (Fig. 1).

Patients undergoing a stand-alone operation had an MIS lateral procedure. This technique, first described as an extreme lateral interbody fusion (L. Pimenta, presented at the Brazilian Spine Society Meeting, 2001), involved the use of a tubular retractor to access the intervertebral disc via a lateral approach through or in front of the psoas muscle. The technique later became even more popular as a direct lateral interbody fusion or MIS lateral interbody fusion. Patients who underwent a stand-alone procedure did not have any posterior surgery, transpedicular screws, or laminectomy. The surgery did involve intervertebral cages, often with an osteobiological fusion material, and possibly with anterior screws to hold the cages in place. In patients in this category surgery was performed at multiple levels in the lumbar spine under a single anesthetic.

For the circumferential MIS group, patients underwent any possible combination of 2 types of minimally invasive fusion. In essence, at any single level of the spine this was either an MIS lateral interbody fusion supplemented with percutaneous pedicle screws at that level or an MIS transforaminal lumbar interbody fusion (TLIF). Typical circumferential MIS procedures would include the following: 1) lumbar lateral interbody fusion supplemented with percutaneous pedicle screws; 2) lateral interbody fusion at multiple levels, with MIS TLIF at levels that are not easily or safely accessible via the lateral approach (L4/5 or L5/S1) and supplemented by percutaneous pedicle screws; 3) multilevel MIS TLIF such as L2$\mathrm{S} 1$; or 4) any of the first 3 surgeries, with extension into the low thoracic spine with percutaneous pedicle screws and MIS facet fusions.

The hybrid group included those patients who had undergone an MIS lateral interbody fusion that was supplemented with an open posterior procedure. The poste- 

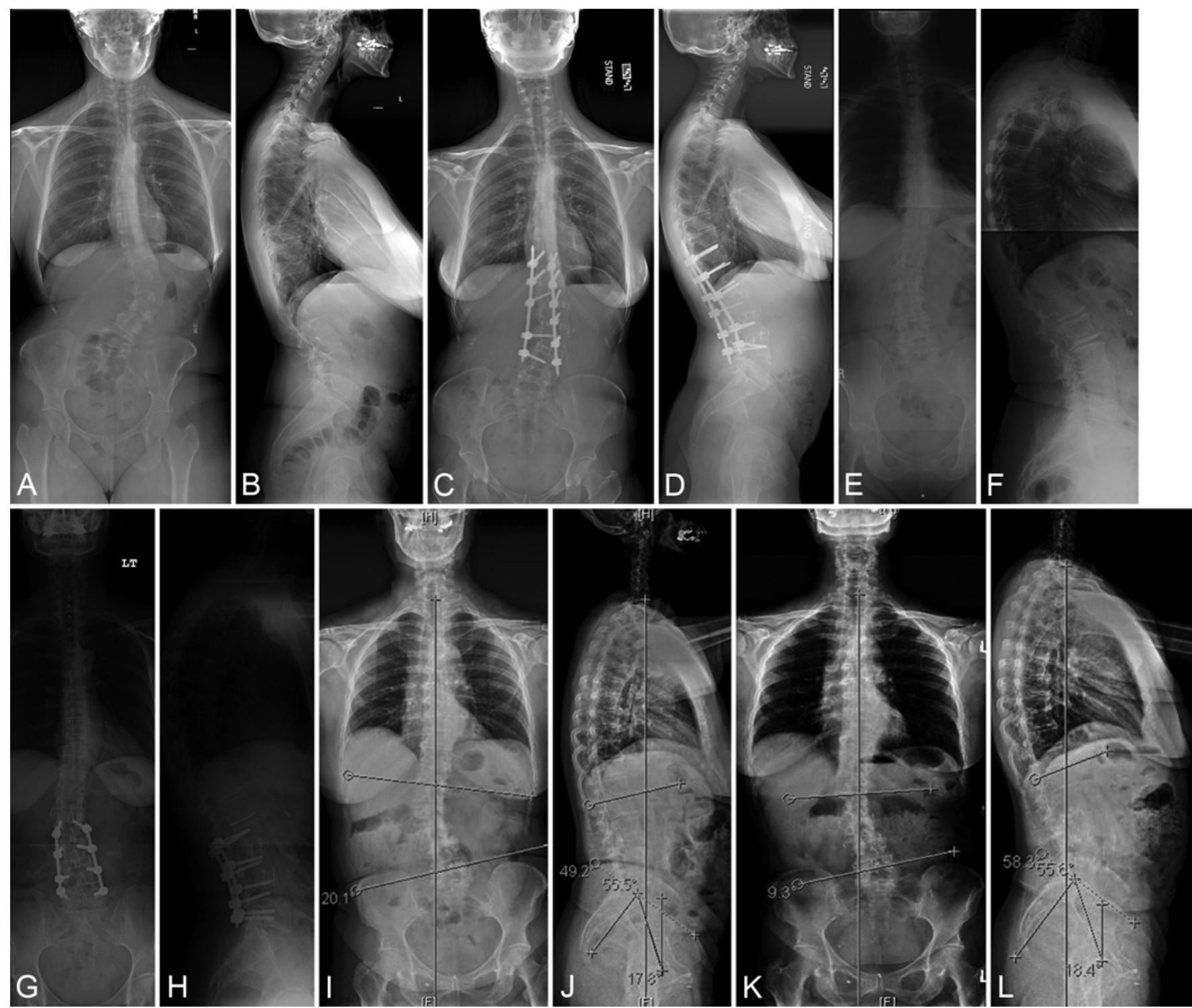

FIG. 1. Radiographs obtained in case examples of the 3 surgical constructs. Preoperative (A and B) and postoperative ( $C$ and D) hybrid constructs. Preoperative (E and F) and postoperative ( $\mathbf{G}$ and $\mathbf{H})$ circumferential MIS constructs. Preoperative (I and J) and postoperative ( $\mathrm{K}$ and $\mathrm{L}$ ) stand-alone constructs.

rior procedure included pedicle screw-rod fixation and in some cases also included osteotomies. Patients with hybrid and circumferential MIS could have undergone a single surgical procedure or one that was staged into 2 anesthetic events.

\section{Results}

\section{Patient Demographic Data}

The MIS-ISSG database included 85 patients who met the inclusion criteria. With regard to surgery type, 7 had a stand-alone procedure, 43 underwent circumferential MIS, and 35 had hybrid surgery. The demographic data for the group are shown in Table 1. For the group as a whole, an average of 4.2 discs (range 3-7) were fused. The mean follow-up was 26.1 months with a minimum of 12 months.

The magnitude of the surgical events was more sub- stantial in the hybrid group and least invasive in the standalone group, with the circumferential MIS group being intermediate. Blood loss and the probability of having the procedures staged into several anesthetic events were also found to be different between the 3 groups (Table 1). Total surgical time was 355,479 , and 748 minutes in the stand-alone, circumferential MIS, and hybrid groups, respectively.

\section{Clinical Outcomes}

Clinical improvement as measured by the NPS and ODI was seen in all 3 groups, as shown in Table 2. Improvements in axial back pain were similar between groups, with $3.0,3.5$, and 4.4 points of improvement seen in the stand-alone, circumferential MIS, and hybrid groups, respectively $(\mathrm{p}>0.05)$. Improvement in leg pain was $4.8,2.3$, and 2.4 points of improvement seen in the 
M. Y. Wang et al.

TABLE 1: Demographic data and surgical parameters in 85 patients with ASD*

\begin{tabular}{lccc}
\hline & & Op Type & Hybrid \\
\cline { 2 - 4 } Characteristic & Stand-Alone & cMIS & 35 \\
\hline no. of patients & 7 & 43 & 65 \\
mean age in yrs & 65 & 64 & $89 \%$ \\
sex (\% female) & $100 \%$ & $77 \%$ & 2.91 \\
mean no. of comorbidities & 4 & $1.72 \dagger$ & 2.2 \\
mean ASA grade & $2.9 \ddagger$ & $2.0 \ddagger$ & $26 \%$ \\
$\%$ w/ prior spine op§ & $57 \%$ & $14 \%$ & 28.4 \\
mean BMl & 30.8 & 25.6 & $6 \%$ \\
$\%$ smokers & $0 \%$ & $21 \%$ & 4.2 \\
mean no. of levels fused & 3.7 & 4.3 & $3-6$ \\
range of levels fused & $3-5$ & $3-7$ & $748 \uparrow$ \\
mean total op time (mins) & 355 & 479 & $1743^{* *}$ \\
mean total surgical blood loss (ml) & 95 & 585 & $89 \%$ \\
$\%$ w/ staged op§ & $0 \%$ & $58 \%$ & $40 \%$ \\
\%/ major complications§ & $29 \%$ & $14 \%$ & $29 \%$ \\
\hline
\end{tabular}

* ASA = American Society of Anesthesiologists; BMI = body mass index; cMIS = circumferential MIS.

$\dagger$ The mean number of comorbidities was significantly lower in the cMIS group compared to the others.

$\ddagger$ The mean ASA grade was significantly lower in the cMIS versus the stand-alone group.

$\S$ The percent with prior spine surgery was significantly different among the groups (we performed a chi-square test with 3 groups, but did not do multiple comparisons). The same comment applies to percent with staged surgery, major complications, and minor complications.

II The mean operating time was significantly longer in the hybrid group compared to the others.

** The mean blood loss during surgery was significantly higher in the hybrid group compared to the others.

stand-alone, circumferential MIS, and hybrid groups, respectively $(\mathrm{p}>0.05)$. Improvement in ODI was similar between groups, with 23.1, 19.5, and 17.7 points of improvement seen in the stand-alone, circumferential MIS, and hybrid groups, respectively $(\mathrm{p}>0.05)$.

Major complications were $29 \%, 14 \%$, and $40 \%$ in the stand-alone, circumferential MIS, and hybrid groups,

TABLE 2: Clinical outcomes in 85 patients with ASD*

\begin{tabular}{lccc}
\hline \multirow{2}{*}{ Outcome } & \multicolumn{3}{c}{ Op Type } \\
\cline { 2 - 4 } NPS & Stand-Alone & cMIS & Hybrid \\
\hline back pain & & & \\
$\quad$ baseline & 6.8 & 6.5 & 7.1 \\
1 yr & 3.8 & 3.0 & 2.7 \\
$\quad$ mean decrease & 3.0 & 3.5 & 4.4 \\
leg pain & & & \\
$\quad$ baseline & 7.8 & 4.6 & 4.8 \\
$\quad$ 1 yr & 3.0 & 2.3 & 2.4 \\
$\quad$ mean decrease & 4.8 & 2.3 & 2.4 \\
ODI $\quad$ & & \\
baseline & & 42.9 & 51.2 \\
1 yr & 48.8 & 23.4 & 33.5 \\
mean decrease & 25.7 & 19.5 & 17.7 \\
\hline
\end{tabular}

* No statistically significant differences were found between groups. respectively $(\mathrm{p}=0.03)$. There were no cases of death, myocardial infarction, or blindness seen in any of the 85 patients. Three $(7 \%)$ of patients who underwent circumferential MIS and $5(14 \%)$ of the patients with hybrid procedures had a return to the operating room.

\section{Radiographic Outcomes (Coronal Plane)}

For the stand-alone group the preoperative Cobb angle ranged from $22^{\circ}$ to $51^{\circ}$, with $57 \%$ of patients having a curve greater than $30^{\circ}$, and $28.6 \%$ greater than $50^{\circ}$. The mean Cobb angle improved from $35.73^{\circ}$ to $30.0^{\circ}$ (p $=0.001)$. A ceiling effect of $23^{\circ}$ for curve correction was observed, regardless of preoperative curve severity or the number of levels treated, as shown in Fig. 2. Cobb angle correction was determined as the difference between the preoperative and last follow-up measurements on 36-in standing radiographs. For the circumferential MIS group the preoperative $\mathrm{Cobb}$ angle ranged from $19^{\circ}$ to $62^{\circ}$, with $44 \%$ greater than $30^{\circ}$ and $5 \%$ greater than $50^{\circ}$. The mean Cobb angle improved from $32^{\circ}$ to $12^{\circ}$ ( $\left.\mathrm{p}<0.001\right)$. A ceiling effect of $34^{\circ}$ for curve correction was observed. For the hybrid group the range for the preoperative Cobb angle was $23^{\circ}-82^{\circ}$, with $74 \%$ greater than $30^{\circ}$ and $23 \%$ greater than $50^{\circ}$. The mean Cobb angle improved from $43^{\circ}$ to $15^{\circ}$. A ceiling effect of $55^{\circ}$ for curve correction was observed $(\mathrm{p}<0.001)$.

\section{Radiographic Outcomes (Sagittal Plane)}

In the sagittal plane for the stand-alone group the 


\section{Ceiling effects for MIS deformity correction}

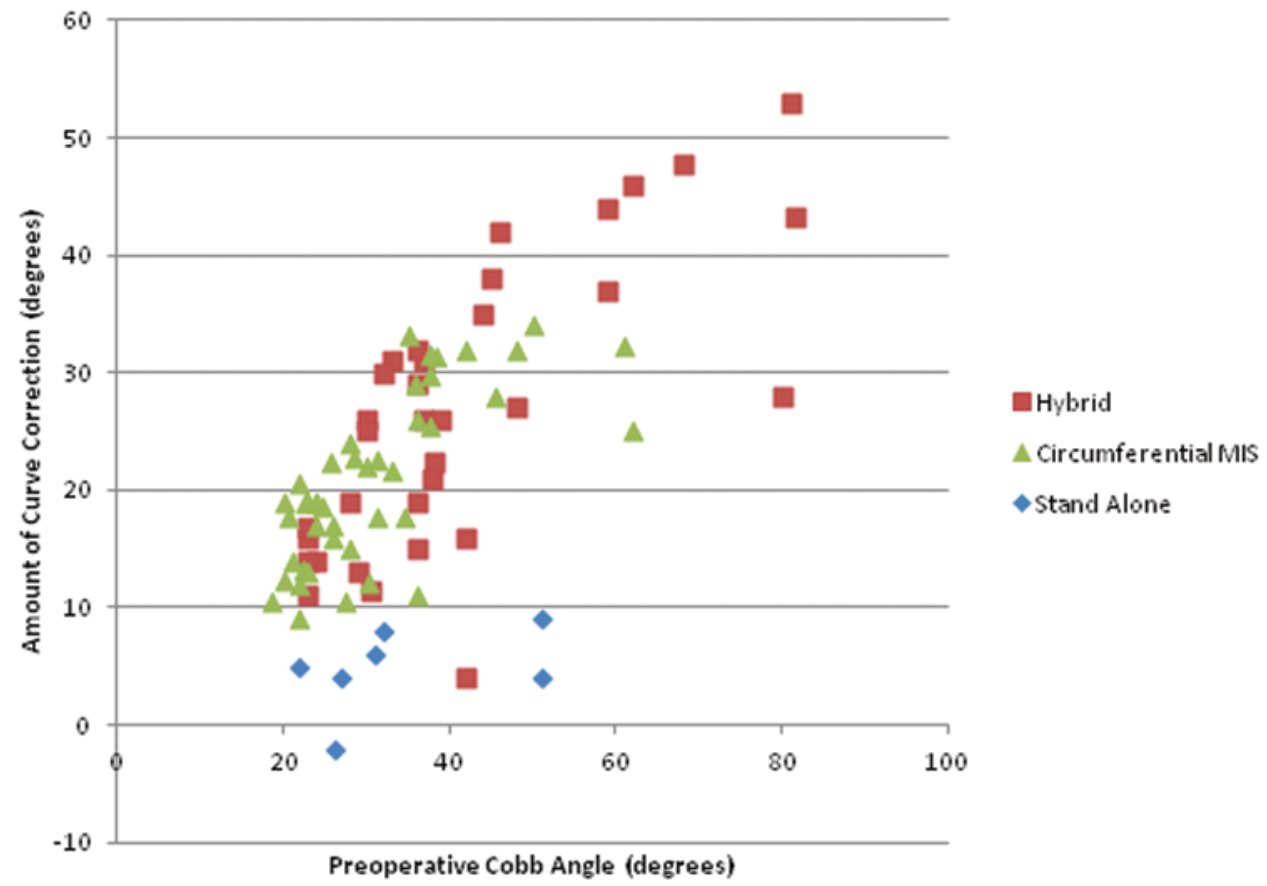

Fig. 2. Scatterplot showing scoliotic curve correction achieved at last follow-up as a function of preoperative curve severity.

preoperative lumbar lordosis averaged $34.7^{\circ} \pm 9.0^{\circ}$, and improved after surgery to an average of $39.7^{\circ} \pm 8.7^{\circ}$. This reflected a mean improvement of $5^{\circ}(p=0.43)$. The preoperative lordosis ranged from $20^{\circ}$ to $48^{\circ}$, and the range at 1 -year follow-up was $27^{\circ}-50^{\circ}$. The preoperative pelvic tilt averaged $33.8^{\circ} \pm 8.2^{\circ}$ and increased after surgery to an average of $36.4^{\circ} \pm 9.5^{\circ}$. This reflected a mean increase of $2.6^{\circ}(\mathrm{p}=0.033)$. The preoperative pelvic tilt ranged from $16.3^{\circ}$ to $42^{\circ}$, and the range at 1 -year follow-up was $20^{\circ}-45^{\circ}$. The preoperative SVA averaged $37.6 \pm 64.8 \mathrm{~mm}$, and after surgery it averaged $37.8 \pm 43.7 \mathrm{~mm}$, reflecting a mean improvement of $20 \mathrm{~mm}(\mathrm{p}=0.9)$. The preoperative SVA ranged from $-8 \mathrm{~mm}$ to $149 \mathrm{~mm}$, and the range at 1-year follow-up was -2 to $109 \mathrm{~mm}$ (Figs. 3 and 4).

In the sagittal plane for the circumferential MIS group the preoperative lumbar lordosis averaged $34.0^{\circ} \pm$ $10.9^{\circ}$, and improved after surgery to an average of $39.6^{\circ}$ $\pm 12.1^{\circ}$. This reflected a mean improvement of $5.7^{\circ}(\mathrm{p}=$ $0.001)$. The preoperative pelvic tilt averaged $26.4^{\circ} \pm 11.5^{\circ}$ and changed minimally after surgery to an average of $25.1^{\circ} \pm 9.1^{\circ}$. This reflected a mean decrease of $1.27^{\circ}(\mathrm{p}=$ 0.41 ). The preoperative SVA averaged $29 \pm 41.4 \mathrm{~mm}$ and after surgery averaged $29.7 \pm 44.6 \mathrm{~mm}$, reflecting a mean increase of $0.8 \mathrm{~mm}(\mathrm{p}=0.889)$.

In the sagittal plane for the hybrid group the preoperative lumbar lordosis averaged $31.9^{\circ} \pm 16.1^{\circ}$, and improved after surgery to an average of $48.5^{\circ} \pm 13.8^{\circ}$. This reflected a mean improvement of $16.6^{\circ}$ ( $\left.\mathrm{p}<0.001\right)$. The preoperative pelvic tilt averaged $34.4^{\circ} \pm 13.7^{\circ}$ and increased after surgery to an average of $34.3^{\circ} \pm 13.7^{\circ}$. This reflected a mean increase of $0.16^{\circ}(\mathrm{p}=0.94)$. The preoperative SVA averaged $62.9 \pm 52.2 \mathrm{~mm}$, and after surgery it averaged $28.7 \pm 24 \mathrm{~mm}$, reflecting a mean improvement of $34.1 \mathrm{~mm}(\mathrm{p}<0.001)$.

\section{Discussion}

The development of new techniques for MIS to treat ASD has been inherently appealing. If spinal MIS procedures can achieve neural decompression, curve correction, and intervertebral fusion with less blood loss and tissue trauma, then these methods may improve patient outcomes by reducing the rate of complications. However, MIS methods are also likely to pose limitations when compared with traditional open surgery. This is expected given that MIS is typically performed over fewer motion segments, and the limited exposures will reduce a surgeon's ability to decompress neural elements, perform osteotomies, manipulate the spinal segments, and fuse exposed dorsal bony surfaces.

To date, most methods have focused on using the MIS lateral transpsoas interbody procedure to assist with correcting the coronal deformity and providing the access for an interbody cage and fusion. This technique, applied with much enthusiasm, has proven to be excellent for treating local degenerative arthritis, restoring foraminal height, achieving indirect neural decompression, and

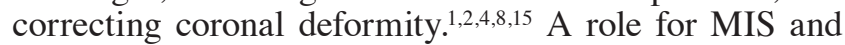
open TLIF has also been seen when performed over multiple motion segments for correcting spinal deformities. ${ }^{7,14}$

\section{Cobb Angle Correction}

However, the ideal application of both MIS TLIF and lateral interbody fusion in the setting of ASD remains unclear. This study was conducted to identify the efficacy of multilevel MIS interbody fusion techniques to correct spinal deformities and achieve clinical improvement. The 3 types of surgery in this study, stand-alone, circumferential MIS, and hybrid procedures, were associated with increasing surgical invasiveness. As expected, 


\section{A Added Lordosis Using Three MIS Techniques}

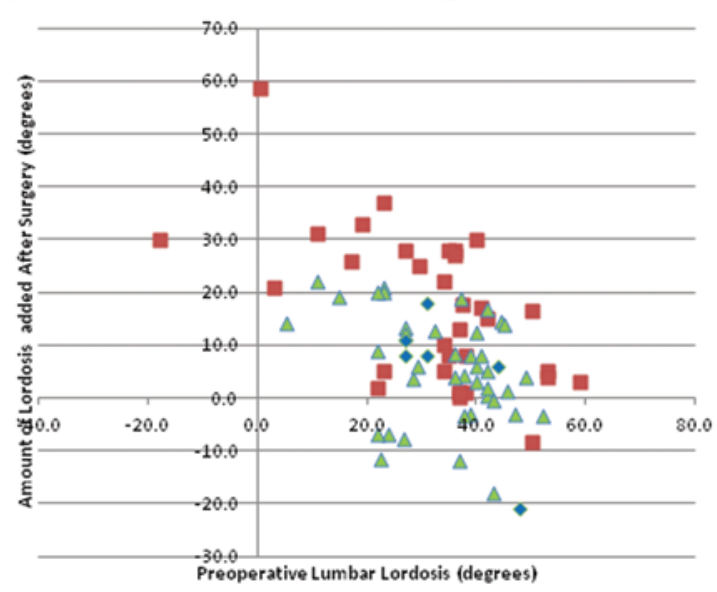

aHybrid

$\triangle$ Circumferential MIS

$\uparrow$ Stand Alone

\section{C}

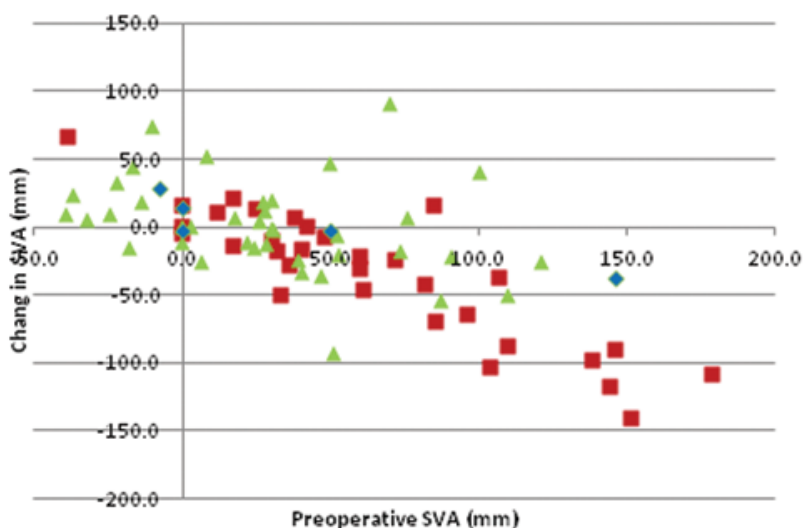

B

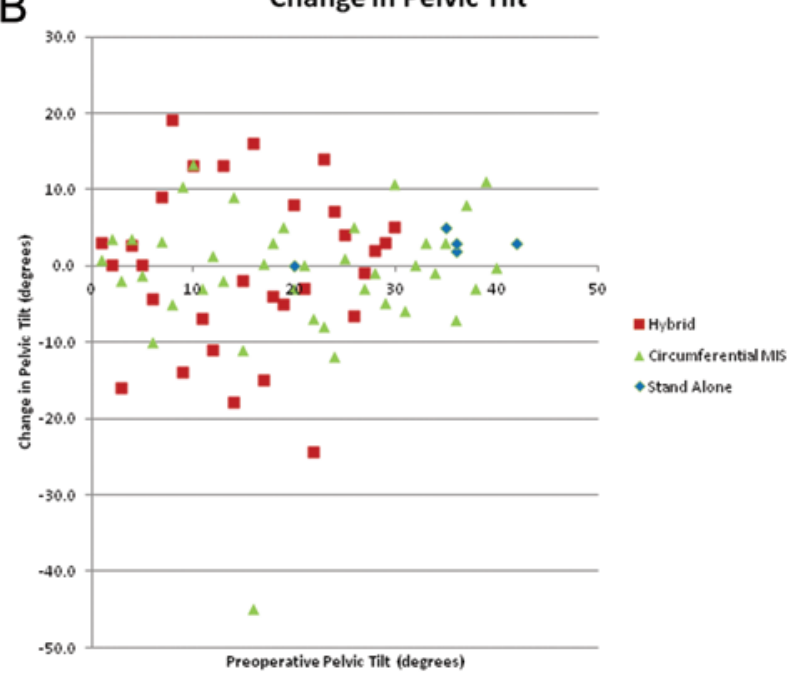

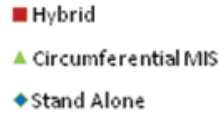

४ Stand Alone

FIG. 3. Scatterplots showing the effects of surgery on deformities in the sagittal plane with respect to lumbar lordosis (A); pelvic tilt (B); and SVA (C).

with increasing invasiveness we saw improvements in the ability to manage more severe scoliotic curves, as well as an improved ability to obtain a good correction. In this study we found a "ceiling effect" with each of the surgical procedures. This was found to be $23^{\circ}, 34^{\circ}$, and $55^{\circ}$ for stand-alone, circumferential MIS, and hybrid techniques, respectively.

Because the stand-alone procedures relied solely on interbody distraction for curve correction and did not involve the use of pedicle screw fixation, it is anticipated that the surgeons used less corrective force and had more difficulty maintaining any curve correction and that patients were susceptible to intervertebral settling. This was reflected in the less robust ability to manage more serious spinal deformities.

However, with increasing invasiveness we also saw an increased amount of blood loss and surgical complications. For example, in the hybrid group, much of the posterior surgery could be considered to be quite similar to an open procedure. This is highlighted by the fact that 14 patients (40\%) in the hybrid group had a Type 3 osteotomy (pedicle subtraction osteotomy) and an associated mean blood loss of $1743 \mathrm{ml}$. These findings highlight the complex decision making and technical maneuvers needed to effect correction of more severe curves, such as those greater than $50^{\circ}$ in the coronal plane.

\section{Sagittal Plane Correction}

The limitations of MIS for deformity were even more apparent when examining spinal deformities in the sagittal plane. In this series the effects of primary interbody surgery (stand-alone and circumferential MIS groups) introduced a minimal amount of lordosis. The average amount of lordosis added at 1 year was $5^{\circ}$ and $5.7^{\circ}$, respectively. The maximal addition of only $22^{\circ}$ of lordosis reveals that these techniques are likely to be of limited long-term benefit for patients with positive sagittal balance as their primary complaint. The hybrid surgeries were able to effect greater improvements in lumbar lordosis, with a maximal addition of $58^{\circ}$. Although the reduction of pain and disability due to neural entrapment, claudication, and disc/facet joint arthritis can be seen when using the less invasive techniques in patients with ASD, these findings should be considered when planning the surgical treatment of patients with a thoracolumbar kyphosis. 


\section{Ceiling effects for MIS deformity correction}

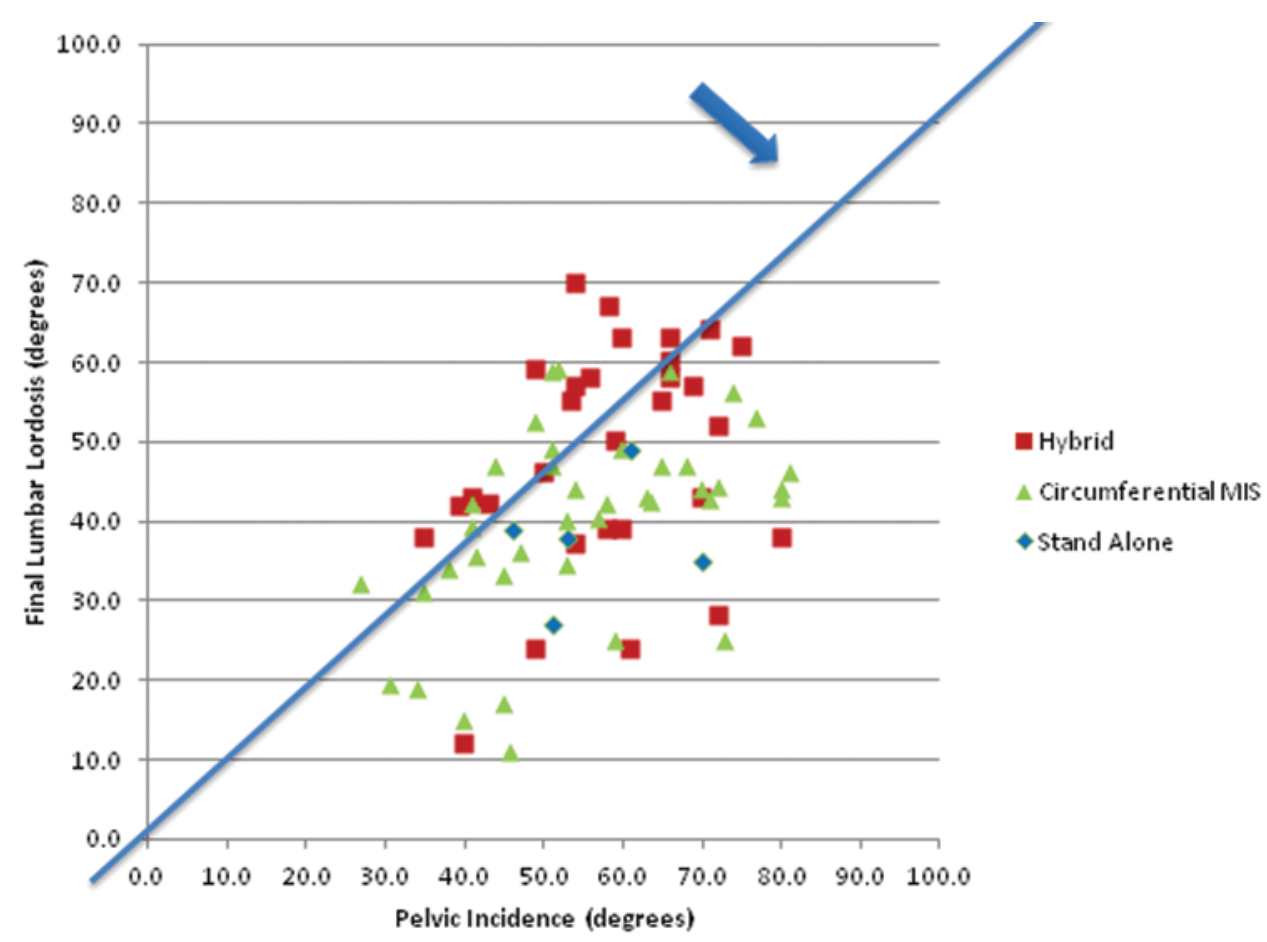

FIG. 4. Scatterplot of final lumbar lordosis in relation to pelvic incidence by procedural subtype.

\section{Clinical Benefits of Surgery}

Any surgical intervention for degenerative spinal disease must consider the entire picture of the individual patient. This includes not only the radiographic findings but also the clinical complaint(s), medical comorbidities, patient expectations, socioeconomic factors, and life expectancy of the patient. In this multicenter clinical study we found that clinical outcomes showed acceptable levels of improvement in all groups, with a mean ODI reduction of 20 points. Although some of the MIS techniques may have been less robust for treating severe deformities, many of the patients in this series did not require a major correction of sagittal or coronal plane abnormalities, as was reflected in their spinal and spinopelvic parameters. Thus, in this series treatments were tailored to the individual patients' needs, with attention paid to avoiding more invasive surgeries if possible. However, the potential implications of undercorrecting a spinal deformity over the longer term is yet another consideration that is beyond the scope of this study.

\section{Complications in MIS}

This study was undertaken by a group of surgeons with specific expertise in minimally invasive spinal surgery. However, in this cohort we still found a significant complication rate. Major complications were seen in $29 \%$, $14 \%$, and $40 \%$ of patients in the stand-alone, circumferential MIS, and hybrid groups, respectively $(\mathrm{p}=0.03)$. Although there were no cases of death, myocardial infarction, or blindness this complication rate remains high, and is similar to those seen with open spinal deformity surgery. In addition, the operating times involved in these interventions was significant, particularly with multistage operations. Thus, surgeons contemplating adoption of these methods should do so with a full understanding of these limitations.

\section{Study Limitations}

This study has several limitations. The first is the classification of all 85 operations into only 3 subtypes. Although there are clear differences between surgeries in the different categories, there is also significant heterogeneity within each of the 3 groups. For example, the circumferential MIS group contained patients who underwent lateral interbody fusion with percutaneous screws, multilevel MIS TLIF, and combination surgeries. Similarly, many patients with hybrid surgery did not have a Level 3 osteotomy, significantly changing the expected blood loss of the dorsal procedure.

Like most retrospective studies, this one is limited by potential underreporting of complications. In addition, with a mean follow-up duration of 26.1 months, this study does not have the ability to ascertain rates of delayed loss of correction, adjacent-level problems, and screw/rod fracture. These are all problems that are well known to occur in a delayed fashion in patients with open ASD.

We enrolled patients at 9 centers with a track record of proficiency in MIS for ASD. However, specific arenas of technical expertise were probably different between sites, and a study of this size would not allow for a meaningful analysis of center effects. Similarly, the selection process for the surgical procedure was uncontrolled. Factors such as a scoliotic curve's flexibility in the coronal plane were not assessed in this study, and this probably has a significant impact on the surgeon's choice of approach. Thus, the ability to correct a patient's Cobb angle is the result of multiple factors, only one of which is the surgical approach taken. 
Finally, the number of cases in the stand-alone group was limited to only 7 patients. This does limit our ability to draw definitive conclusions as to the utility of this surgical procedure.

\section{Conclusions}

This study is the first to our knowledge to compare a multitude of MIS methods for ASD to assess their efficacy. Although there are numerous study limitations, it does appear that the choice of surgical approach can impose specific limitations on the surgeon's ability to correct a spinal deformity. Furthermore, as expected, the less invasive approaches were associated with a more limited ability to correct severe curves, whereas more invasive strategies were able to achieve results comparable to open surgery. Proper clinical decision making resulted in acceptable clinical outcomes in all groups.

\section{Disclosure}

Dr. Wang is a consultant for and also holds a patent from DePuy Spine. Dr. Mummaneni has received honoraria from Globus; royalties from DePuy Spine, quality Medical Publishers, and Thieme; and owns stock in Spinicity. Dr. Fu is a consultant for Medtronic and has received an honorarium from DePuy Spine. Dr. Anand is a patent holder with Medtronic and is also a consultant for that company and for Baxano Surgical. Dr. Okonkwo receives royalties from Lanx. Dr. Kanter receives royalties from Lanx, and from NuVasive he has received support of non-study-related clinical or research effort that he oversaw. Dr. La Marca is a consultant for Globus and for Biomet. Dr. Fessler owns In Queue Innovations and is a consultant for DePuy Synthes. Dr. Uribe is a consultant for NuVasive and Orthofix. Dr. Shaffrey is a consultant for the following companies: Biomet, Globus, Medtronic, NuVasive, and Stryker. He also is a patent holder with and receives royalties from Biomet and Medtronic. Dr. Deviren is a consultant for NuVasive, Guidepoint, and Stryker. Dr. Mundis is a consultant for $\mathrm{K} 2 \mathrm{M}$ and $\mathrm{NuVasive.} \mathrm{He} \mathrm{received} \mathrm{support} \mathrm{from}$ NuVasive for a non-study-related clinical or research effort that he oversaw, and he also received research support from ISSGF, as well as fellowship support from NuVasive, Pioneer, and the Orthopaedic Research and Education Foundation (OREF).

Author contributions to the study and manuscript preparation include the following. Conception and design: Wang, Mummaneni, Uribe. Acquisition of data: Wang, Mummaneni, Anand, Okonkwo, Kanter, La Marca, Fessler, Haque, Deviren, Mundis. Analysis and interpretation of data: Wang, Fu, Uribe, Lafage. Drafting the article: Wang, Mummaneni, Mundis. Critically revising the article: Wang, Okonkwo, La Marca, Shaffrey, Mundis. Reviewed submitted version of manuscript: Wang, Mummaneni. Approved the final version of the manuscript on behalf of all authors: Wang. Statistical analysis: Wang, Lafage. Administrative/technical/material support: Wang. Study supervision: Wang, Shaffrey.

\section{References}

1. Anand N, Baron EM, Thaiyananthan G, Khalsa K, Goldstein TB: Minimally invasive multilevel percutaneous correction and fusion for adult lumbar degenerative scoliosis: a technique and feasibility study. J Spinal Disord Tech 21:459-467, 2008
2. Anand N, Rosemann R, Khalsa B, Baron EM: Mid-term to long-term clinical and functional outcomes of minimally invasive correction and fusion for adults with scoliosis. Neurosurg Focus 28(3):E6, 2010

3. Bess S, Boachie-Adjei O, Burton D, Cunningham M, Shaffrey C, Shelokov A, et al: Pain and disability determine treatment modality for older patients with adult scoliosis, while deformity guides treatment for younger patients. Spine (Phila Pa 1976) 34:2186-2190, 2009

4. Dakwar E, Cardona RF, Smith DA, Uribe JS: Early outcomes and safety of the minimally invasive, lateral retroperitoneal transpsoas approach for adult degenerative scoliosis. Neurosurg Focus 28(3): E8, 2010

5. Fairbank JC: Why are there different versions of the Oswestry Disability Index? J Neurosurg Spine 20:83-86, 2014

6. Fehlings MG, Ibrahim GM: Editorial. Spinal deformity. J Neurosurg Spine 13:663-665, 2010

7. Heary RF, Karimi RJ: Correction of lumbar coronal plane deformity using unilateral cage placement. Neurosurg Focus 28(3):E10, 2010

8. Isaacs RE, Hyde J, Goodrich JA, Rodgers WB, Phillips FM: A prospective, nonrandomized, multicenter evaluation of extreme lateral interbody fusion for the treatment of adult degenerative scoliosis: perioperative outcomes and complications. Spine (Phila Pa 1976) 35 (26 Suppl):S322-S330, 2010

9. Mummaneni PV, Dhall SS, Ondra SL, Mummaneni VP, Berven S: Pedicle subtraction osteotomy. Neurosurgery 63 (3 Suppl):171-176, 2008

10. Pateder DB, Gonzales RA, Kebaish KM, Cohen DB, Chang JY, Kostuik JP: Short-term mortality and its association with independent risk factors in adult spinal deformity surgery. Spine (Phila Pa 1976) 33:1224-1228, 2008

11. Scheufler KM, Cyron D, Dohmen H, Eckardt A: Less invasive surgical correction of adult degenerative scoliosis, part I: technique and radiographic results. Neurosurgery 67:696710,2010

12. Schwender JD, Holly LT, Rouben DP, Foley KT: Minimally invasive transforaminal lumbar interbody fusion (TLIF): technical feasibility and initial results. J Spinal Disord Tech 18 Suppl:S1-S6, 2005

13. Smith JS, Shaffrey CI, Glassman SD, Berven SH, Schwab FJ, Hamill CL, et al: Risk-benefit assessment of surgery for adult scoliosis: an analysis based on patient age. Spine (Phila Pa 1976) 36:817-824, 2011

14. Wang MY: Improvement of sagittal balance and lumbar lordosis following less invasive adult spinal deformity surgery with expandable cages and percutaneous instrumentation. Clinical article. J Neurosurg Spine 18:4-12, 2013

15. Wang MY, Mummaneni PV: Minimally invasive surgery for thoracolumbar spinal deformity: initial clinical experience with clinical and radiographic outcomes. Neurosurg Focus 28(3):E9, 2010

Manuscript submitted January 25, 2014.

Accepted March 25, 2014.

Please include this information when citing this paper: DOI: 10.3171/2014.3.FOCUS1423.

Address correspondence to: Michael Y. Wang, M.D., Department of Neurological Surgery, Lois Pope Life Center, 1095 NW 14th Terrace, Miami, FL 33136. email: MWang2@med.miami.edu. 